\title{
Plataforma simuladora de reabsorções radiculares internas para pesquisa endodôntica
}

\author{
A plataforma simuladora de reabsorções internas representa mais \\ um importante recurso para o ensino da Endodontia pré-clínica.
}

Carlos Alberto de Campos*, Celso Neiva Campos**

* Professor Livre-Docente da Faculdade de Odontologia da

Universidade Federal de Juiz de Fora (UFJF) - MG.

** Professor Adjunto Doutor Coordenador do Curso de

Especialização em Endodontia da FO - UFJF - MG.

\section{RESUMO}

O presente trabalho objetiva a possibilidade do preparo de cavidades no interior dos canais radiculares, simulando reabsorções internas. Em pesquisas por nós desenvolvidas, sentimos dúvidas quanto aos resultados obtidos, porquanto as cavidades tinham forma e volume muito diferentes. Decidimos, então, estudar e projetar um aparelho que permitisse o preparo de cavidades com a máxima semelhança em diâmetro e no sentido longitudinal. Assim, idealizamos a plataforma simuladora, em que um dente natural ou artificial é preso a uma base que se movimenta no sentido longitudinal da plataforma. Esta base encontra-se em frente a um conjunto móvel, que sustenta uma peça de mão (turbina) para baixa rotação, à qual é adaptada uma broca esférica de $28 \mathrm{~mm}$, \# 1, \# 2 ou \# 3, para girar a $200 \mathrm{rpm}$, com sua haste cônica transformada em cilíndrica por desgaste no esmeril, tendo sua parte ativa (esfera) torcida para lateral, depois de aquecida ao rubro. Através do movimento da base em direção ao conjunto móvel, acontece o acoplamento dente-broca. Este conjunto, que sustenta a turbina, movimenta-se numa escala milimetrada, apenas no sentido transversal ao eixo longitudinal da plataforma, permitindo mover a broca para lateral, o que produz um aumento no diâmetro da cavidade interna. Concluímos que a plataforma simuladora constitui real contribuição técnica ao ensino pré-clínico da Endodontia, bem como a pesquisadores no estudo dos sistemas de irrigação, remoção do "smear layer" e preenchimento de reabsorções internas por diferentes materiais e técnicas de obturação.

\section{DESCRITORES}

Pesquisa em Odontologia. Endodontia. Reabsorção de dente.

É de importância indiscutível o trabalho pré-clínico para o aprendizado endodôntico e o desenvolvimento de pesquisas sobre instrumentação e obturação dos canais radiculares (QUALTROUGH $e t$ al., 1999; Wesselink, 1991; PETERS, BARbAKOW, 2000). Se utilizamos dentes de humanos, todas as etapas se realizam naturalmente, sem dificuldades relativas à anatomia interna do canal que não sejam idênticas às que se apresentam in vivo. No entanto, quando o objetivo é efetuar pesquisa sobre tratamento em raízes com reabsorção interna, torna-se necessário o exame da cavidade, pós-tratamento, para averiguação dos resultados, seja direta ou indiretamente (HASSELGREN, STRÖMBERG, 1976; LYROUDIA et al., 2002). Assim, na observação sobre diferentes técnicas para preenchimento por obturações, ou sobre desinfecção, remoção de "smear layer" e outros temas de importância relevante para o estudo da Endodontia, temos que visualizar o que aconteceu dentro da cavidade. Portanto, logicamente, ela terá que existir.

Realizamos trabalho (CAMPOS, CAMPOS, 2003) em que idealizamos um método para simulação que nos permitiu chegar a resultados interessantes, cuja análise estatística demonstrou ser de importância clínica, mas que nos levou também a pensar sobre a possibilidade de simular reabsorções o mais idênticas possíveis. 


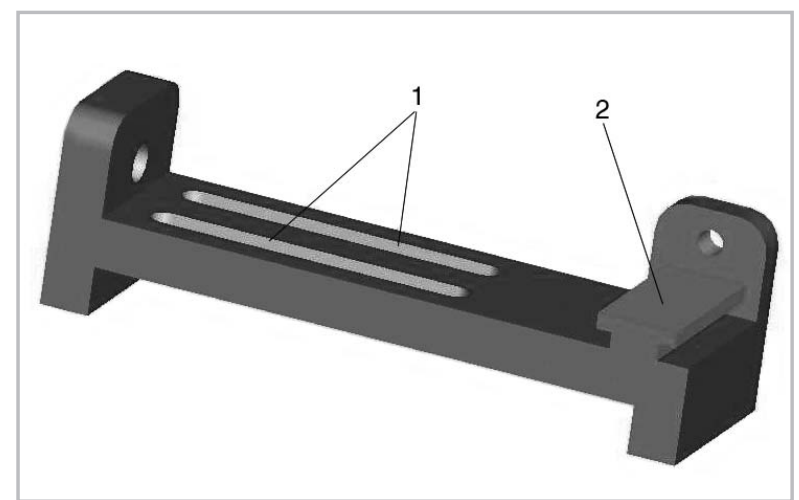

Figura 1 - Peça denominada plataforma, tendo no sentido longitudinal duas canaletas vazadas (1) e transversalmente uma peça tipo gaveta (2).

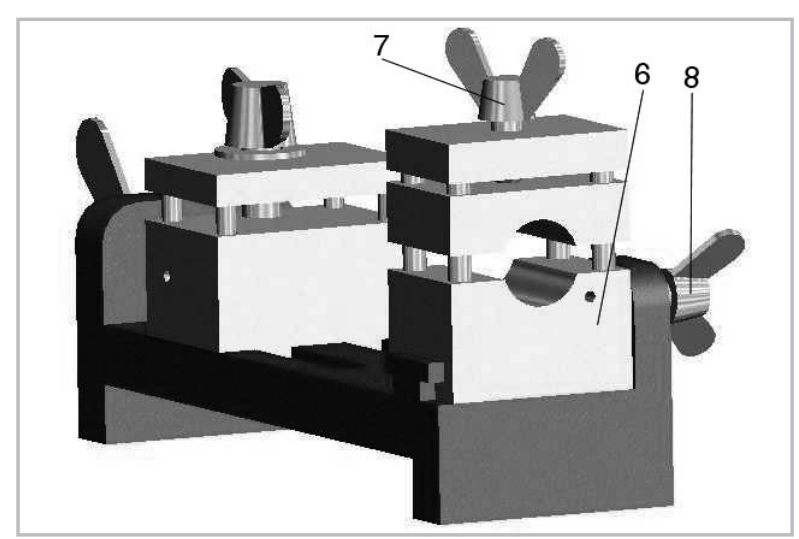

Figura 3 - Conjunto (6) em que será adaptada a turbina de baixa rotação pela torção do parafuso superior (7). O movimento horizontal transversal do conjunto é feito pelo deslizamento na gaveta por torção do parafuso horizontal (8).

Resolvemos, então, aprimorar nossa idealização. As reabsorções que eram efetuadas manualmente nas outras pesquisas, são agora preparadas mecanicamente, através da Plataforma Simuladora, possibilitando resultados mais convincentes.

\section{MATERIAL E MÉTODOS}

Partimos de uma peça metálica - plataforma (Figura 1), confeccionada em alumínio, a fim de baratear o custo do produto, tendo no sentido longitudinal duas canaletas paralelas, vazadas, a fim de possibilitar o movimento de uma base (Figura 2), de mesmo material, com a finalidade de apreender um dente natural ou artificial e se movimentar longitudinalmente, em direção a uma broca esférica, de $28 \mathrm{~mm}$, cuja parte ativa foi levada à chama de uma lamparina, aquecida ao rubro e torcida em ângulo aproximado de 75 graus. A apreensão do dente é feita pela rotação de um parafuso superior, tipo borboleta, que produz movimento vertical da parte supe-

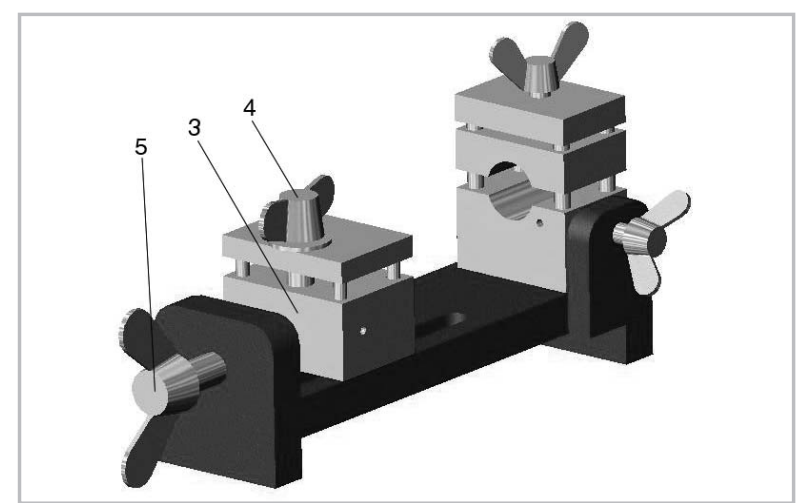

Figura 2 - Base para apreensão de dentes (3) pela pressão vertical de um parafuso (4), adaptada às canaletas para movimentação horizontal pela torção de um parafuso (5).

rior da base, através de quatro pinos posicionados nas extremidades da mesma base. Numa das extremidades da plataforma (Figura 1), colocamos uma peça transversal, tipo gaveta, para proporcionar a movimentação de um conjunto (Figura 3) no sentido da mesma peça, pela torção de um parafuso tipo borboleta, situado lateralmente. O conjunto fará a apreensão de uma turbina de baixa rotação, movida a ar comprimido ou elétrica, pela torção de um parafuso tipo borboleta, superior, que fará a parte superior do conjunto deslizar verticalmente através de quatro pinos situados nas extremidades. Tendo adaptada a turbina (Figura 4), nela colocamos a broca especial. A base com o dente irá se movimentar longitudinalmente à plataforma até a penetração da broca atingir o limite pré-fixado por uma odontometria. A broca, colocada em movimento horário, com aproximadamente $200 \mathrm{rpm}$, poderá ser desviada horizontalmente para aumentar o diâmetro da reabsorção simulada, milimetricamente, através do deslizamento do conjunto na gaveta (Figura 5). Tendo sido registrado o desvio da broca e a odontometria, teremos condições para reproduzir reabsorções.

\section{CONCLUSÕES}

A plataforma simuladora de reabsorções internas representa mais um recurso para o estudo da Endodontia pré-clínica nas Faculdades de Odontologia, nos cursos de aperfeiçoamento e para o desenvolvimento de pesquisas endodônticas, apresentando-se como:

1. Capaz de preparar diversas cavidades internas semelhantes, em dentes naturais ou artificiais, simulando reabsorções.

2. Uma contribuição ao estudo de técnicas de instrumentação, de irrigação/aspiração, de remoção 


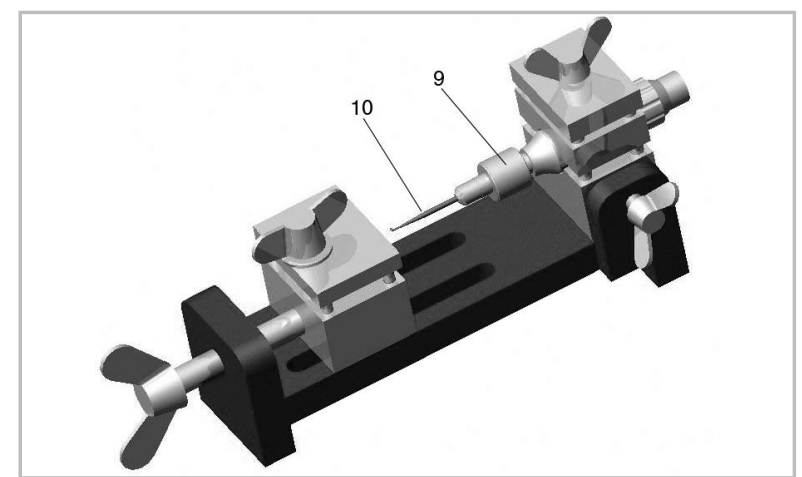

Figura 4 - Turbina adaptada (9), com a broca especial (10).

de "smear layer" e de obturação do sistema de canais radiculares.

3. Um produto de baixo custo, porquanto pode ser fabricado totalmente em blocos de alumínio.

\section{ABSTRACT}

\section{A simulator platform for internal radicular resorptions in endodontic research}

The aim of the present work was to determine the possibility of preparing cavities inside root canals to simulate internal resorptions. While conducting research, doubts emerged regarding the results, because the cavities had markedly different shapes and volumes. We then decided to study and design a device to permit cavity preparation with the maximum possible similarity in diameter and longitudinal direction. So, we conceived the Simulator Platform, wherein a natural or artificial tooth is held in a base that moves longitudinally along the platform. This base is in front of a movable set that supports a low-speed hand-piece (turbine), to which is adapted a spherical \#1, \#2 or \#3 $28 \mathrm{~mm}$ drill, operating at $200 \mathrm{rpm}$, with its conical stem transformed into a cylinder by emery detrition, and its active part (sphere) twisted laterally, after being heated until red hot. The tooth-drill coupling occurs by means of the base movement toward the movable set. This set, which supports the turbine, moves on a millimeter scale, only in the transversal direction on the longitudinal axis of the platform, to make it possible to move the drill laterally, which creates an enlargement in the

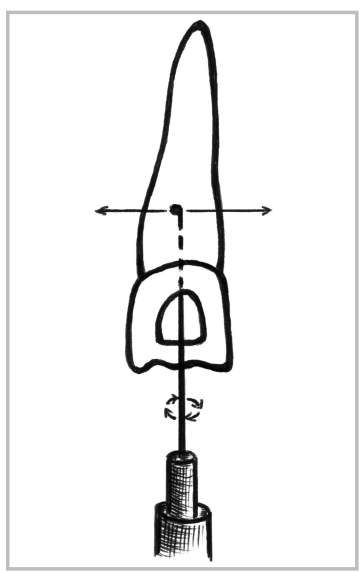

Figura 5 - Broca especial girando internamente em dente natural e movimentando-se também no sentido horizontal junto com a turbina de baixa rotação.

diameter of the internal cavity. We concluded that the Simulator Platform is a real technical contribution to preclinic teaching of endodontics, as well as to researchers in the studies of irrigation systems, smear-layer removal and filling of internal resorptions with different materials and obturation techniques.

\section{DESCRIPTORS}

Dental research. Endodontics. Tooth resorption.

\section{REFERÊNCIAS BIBLIOGRÁFICAS}

CAMPOS, C. N.; CAMPOS, C. A. Comparação de três técnicas de obturação no preenchimento de reabsorções internas. Rev Bras Odontol, v. 60, n. 3, p. 164-166, mai./jun. 2003.

HASSELGREN, G.; STRÖMBERG, T. Histochemical demonstration of acid hydrolase activity in internal dentinal resorption. Oral Surg Oral Med Oral Pathol, v. 42, n. 3, p. 381-385, Sept. 1976.

LYROUDIA, K. M. et al. Internal root resorption studied by radiography, stereomicroscope, scanning electron microscope and computerized $3 \mathrm{D}$ reconstructive method. Dent Traumatol, v. 18, n. 3, p. 148-152, June 2002.

PETERS, O. A.; BARBAKOW, F. Effects of irrigation on debris and smear layer on canal walls prepared by two rotary techniques: a scanning electron microscope study. J Endod, v. 26, n. 1, p. 6-10, Jan. 2000.

QUALTROUGH, A. J.; WhITWORTH, J. M.; DUMMER, P. M. Preclinical endodontology: an international comparison. Int Endod J, v. 32, n. 5, p. 406-414, Sept. 1999.

WESSELINK, P. R. The curriculum in endodontology at the Academic Centre for Dentistry Amsterdam (ACTA), the Netherlands. Int Endod J, v. 24, n. 4, p. 161-168, July 1991.

Aceito para publicação em 11/2003 